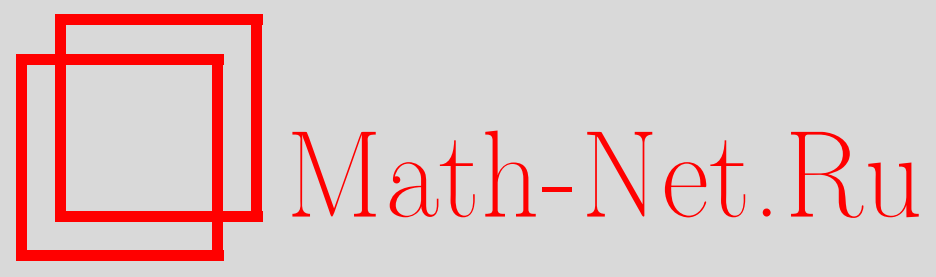

О. В. Денисов, Локальная предельная теорема для распределения части спектра случайной двоичной функции, Дискрет. матем., 2000, том 12, выпуск 1, 82-95

DOI: https://doi.org/10.4213/dm314

Использование Общероссийского математического портала Math-Net.Ru подразумевает, что вы прочитали и согласны с пользовательским соглашением http://www.mathnet.ru/rus/agreement

Параметры загрузки:

IP: 35.173 .137 .237

26 апреля 2023 г., 15:08:59

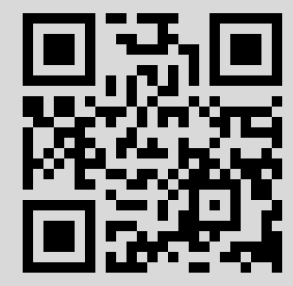


УДК 519.7

\title{
Локальная предельная теорема для распределения части спектра случайной двоичной функции
}

(C) 2000 г. О.В. Денисов

\begin{abstract}
Доказана локальная предельная теорема для распределения вектора растущей размерности, состоящего из некоторых спектральных коэффициентов случайной двоичной функции от $n$ переменных. Исправлена ошибка, допущенная в асимптотической формуле для числа корреляционно-иммунных порядка $k$ функций в одной из статей автора. Получена асимптотическая формула для числа $(n, 1, k)$-устойчивых функций при $k=k(n)=o(\sqrt{n})$.
\end{abstract}

\section{1. Введение}

Пусть функция $f$ выбирается случайно и равновероятно из множества $B_{n}$ всех двоичных функций от $n$ переменных. Это эквивалентно независимому равновероятному выбору ее значений $f(\alpha) \in\{0,1\}$ для всех $\alpha$ из множества $V_{n}$ двоичных векторов длины $n$. Для произвольного подмножества $I=\left\{i_{1}, \ldots, i_{|I|}\right\}$ множества $\{1, \ldots, n\}$ через $w_{I}$ обозначим вес $\left\|f_{i_{1}, \ldots, i_{|I|}}^{1, \ldots, 1}\right\|$ подфункции $f_{i_{1}, \ldots, i_{|I|}}^{1, \ldots, 1}$ функции $f$, получаемой, если в функции $f$ координаты с номерами $i_{1}, \ldots, i_{|I|}$ положить равными единице. Таким же способом будем индексировать другие случайные величины (с. в.) и переменные, полагая, что $I$ принимает значения подмножеств множества $\{1, \ldots, n\}$ мощности от 0 до $k$. Если в формуле не указаны пределы изменения $I$, то подразумевается, что $I$ принимает все значения из $I$ в лексикографическом порядке.

Как в [1], определим случайный вектор (сл.в.)

$$
\bar{w}=\bar{w}(f, n, k)=\left(w_{I}, 0 \leqslant|I| \leqslant k, I \subset\{1, \ldots, n\}\right)
$$

длины

$$
M=M(n, k)=\left(\begin{array}{l}
n \\
0
\end{array}\right)+\ldots+\left(\begin{array}{l}
n \\
k
\end{array}\right) .
$$

При $k=1$ вектор $\bar{w}$ можно представить как

$$
\bar{w}(f, n, 1)=\sum_{x \in V_{n}}\left(1, x_{1}, \ldots, x_{n}\right) f(x)=\left(\|f\|, \sum_{x \in f^{-1}(1)}\left(x_{1}, \ldots, x_{n}\right)\right) .
$$


В таком виде он был введен в работе Чоу [6], в которой было доказано, что любая пороговая функция однозначно определяется этим вектором. С тех пор параметры Чоу являются одной из важнейших характеристик пороговых функций (см. [2]).

В [1] для произвольного фиксированного $k$ был доказан ряд лемм, фактически определяющих предельное распределение $\bar{w}$, и получена асимптотическая формула для мощности класса $K(n, k)$ корреляционно-иммунных порядка $k$ двоичных функций от $n$ переменных. Как будет показано ниже, в результате ошибки автора в нее не был включен возникающий при $k \geqslant 2$ сомножитель

$$
\left(1+\sum_{i=2}^{k}(i-1)^{2}\left(\begin{array}{c}
n \\
i
\end{array}\right)\right)^{-1 / 2}
$$

В данной статье по схеме, использовавшейся в [1], доказывается локальная предельная теорема (ЛПТ) для распределения $\bar{w}$ при произвольном $k$, зависящем от $n$ так, что $k(n)=o(\sqrt{n})$ при $n \rightarrow \infty$. Новые обозначения и идеи позволили существенно упростить формулировки и доказательства многих лемм. Из ЛПТ в качестве следствий выводятся асимптотические формулы для мощности класса $K(n, k)$ и класса

$$
R(n, k)=K(n, k) \cap\left\{f \in B_{n}:\|f\|=2^{n-1}\right\}
$$

$(n, 1, k)$-устойчивых функций, которые в двоичном случае представляют собой уравновешенные корреляционно-иммунные порядка $k$ функции. Приближенные значения, вычисленные по этим формулам, сравниваются с точными значениями, найденными автором с помощью ЭВМ для $n=4,5, k=1,2$. Различные свойства корреляционно-иммунных и устойчивых функций изучались в ряде работ зарубежных авторов (см. обзор [3]).

Порядок корреляционной иммунности можно определить по вектору

$$
\bar{F}=\bar{F}(f, n, k)=\left(F_{I}, 0 \leqslant|I| \leqslant k, I \subset\{1, \ldots, n\}\right),
$$

состоящему из первых (то есть соответствующих векторам веса от 0 до $k$ ) спектральных коэффициентов Фурье-Уолша-Адамара

$$
\begin{aligned}
F_{I} & =\frac{1}{2} \sum_{x \in V_{n}}(-1)^{f(x)+x_{i_{1}}+\ldots+x_{i_{|I|}}}=2^{n-1}-\left\|f(x) \oplus x_{i_{1}} \ldots \oplus x_{i_{|I|}}\right\| \\
& =2^{n-1} \operatorname{Ind}\{I=\varnothing\}-\sum_{x \in V_{n}}(-1)^{x_{i_{1}}+\ldots+x_{i_{|I|}}} f(x) .
\end{aligned}
$$

Здесь $\operatorname{Ind}(A)$ - индикатор события $A$.

ЛПТ для распределения фиксированного числа некоторых спектральных коэффициентов случайной двоичной функции была доказана в работах Б.В. Рязанова и С.И. Чечеты $[4,5]$. В данной статье будет получена ЛПТ для распределения вектора $\bar{F}$ экспоненциально растущей размерности $M(n, k)$.

\section{2. ЛПТ для распределения вектора весов}

В следующей теореме фактически утверждается, что распределение сл.в. $\bar{w}$ можно приблизить плотностью соответствующего $M$-мерного нормального распределения. Здесь и далее $\exp _{2} x=2^{x}, \mathbf{E} \bar{w}$ - математическое ожидание сл.в. $\bar{w}, Q^{T}-$ матрица, полученная транспонированием матрицы $Q, \operatorname{det} Q-$ определитель $Q$. 
Теорема 1. Пусть $n \rightarrow \infty, k(n)=o(\sqrt{n}), Q=Q(n, k)-$ ковариационная матрица сл.в. $(\bar{w}-\mathbf{E} \bar{w}) / 2^{n / 2-1}, \bar{z}(n) 2^{n / 2-1}$ - последовательность целочисленных векторов размерности $M(n, k)$. Тогда равномерно относителъно $\bar{z}$

$$
\begin{aligned}
\mathbf{P}\left\{\bar{w}=\mathbf{E} \bar{w}+\bar{z}(n) 2^{n / 2-1}\right\} & =\frac{\exp \left(-(1 / 2) \bar{z}^{T} Q^{-1} \bar{z}\right)+O\left(n^{5 k+4} / 2^{n}\right)}{\left(2^{n / 2-1}\right)^{M} \sqrt{(2 \pi)^{M} \operatorname{det} Q}} \\
& =\frac{\exp \left(-(1 / 2) \sum_{I}\left(2^{|I|} z_{I}-\sum_{i \in I} 2^{|I|-1} z_{I \backslash\{i\}}\right)^{2}\right)+O\left(n^{5 k+4} / 2^{n}\right)}{\exp _{2}\left(\left(\begin{array}{l}
n \\
k
\end{array}\right)(n-k) / 2+M(n, k) \log _{2} \sqrt{\pi / 2}\right)} .
\end{aligned}
$$

Доказательство. Очевидно,что

$$
\begin{aligned}
w_{I} & =\sum_{\alpha \in V_{n}} \beta_{I}(\alpha) f(\alpha), \\
\mathbf{E} w_{I} & =2^{n-1-|I|} \\
w_{I}-\mathbf{E} w_{I} & =\sum_{\alpha \in V_{n}} \beta_{I}(\alpha)(f(\alpha)-1 / 2),
\end{aligned}
$$

где $\beta_{I}(\alpha)=\alpha_{i_{1}} \ldots \alpha_{i_{|I|}}$ (при $I=\varnothing$ считаем, что $\beta_{I}(\alpha) \equiv 1$ ). Тогда для векторов

$$
\beta(\alpha)=\left(\beta_{I}(\alpha), 0 \leqslant|I| \leqslant k\right), \quad \xi(\alpha)=\beta(\alpha)(f(\alpha)-1 / 2), \quad \xi=\bar{w}-\mathbf{E} \bar{w}
$$

справедливо соотношение

$$
\xi=\sum_{\alpha \in V_{n}} \xi(\alpha)
$$

Характеристические функции случайных векторов $\xi(\alpha)$ и $\xi$ равны соответственно

$$
\varphi_{\alpha}(t)=\cos (\beta(\alpha), t / 2), \quad \varphi_{\xi}(t)=\prod_{\alpha \in V_{n}} \varphi_{\alpha}(t)
$$

где $(x, y)$ - скалярное произведение векторов $x, y$. Умножив равенство

$$
\varphi_{\xi}(t)=\sum_{a \in Z^{M}} \mathbf{P}(\xi=a) \exp (-i(t, a))
$$

на $\exp \left(-i\left(t, z 2^{n / 2-1}\right)\right)$ и проинтегрировав его по переменным $t_{I}, 0 \leqslant|I| \leqslant k$, в пределах от $-\pi$ до $\pi$, находим, что

$$
(2 \pi)^{M} \mathbf{P}\left(\xi=z 2^{n / 2-1}\right)=\int_{-\pi}^{\pi} \ldots \int_{-\pi}^{\pi} \varphi_{\xi}(t) \exp \left(-i\left(t, z 2^{n / 2-1}\right)\right) d t .
$$

Делая замену $x=t 2^{n / 2-1}$ и обозначая через $G$ гиперкуб $\left[-2^{n / 2} \pi / 2,2^{n / 2} \pi / 2\right]^{M}$, получаем, что

$$
\begin{aligned}
\left(\pi 2^{n / 2}\right)^{M} \mathbf{P}\left(\xi=z 2^{n / 2-1}\right) & =\int_{G} \varphi_{\xi}\left(x / 2^{n / 2-1}\right) \exp (-i(x, z)) d x \\
\varphi_{\xi}\left(x / 2^{n / 2-1}\right) & =\prod_{\alpha} \cos \left(\beta(\alpha), x / 2^{n / 2}\right)
\end{aligned}
$$


Для $i=0,1, \ldots, k$ определим функцию

$$
\Psi(n, i)=\left(\left(\begin{array}{l}
n \\
i
\end{array}\right) 2^{n / 3+k i}\right)^{-1}
$$

и разобьем $G$ на попарно не пересекающиеся области

$$
\begin{aligned}
& G_{i}=\left\{x \in G: \exists I:|I|=i,\left|x_{I} / 2^{n / 2}\right|>\Psi(n,|I|) ; \forall I:|I| \geqslant i+1,\left|x_{I} / 2^{n / 2}\right| \leqslant \Psi(n,|I|)\right\}, \\
& G^{*}=G \backslash\left(\bigcup_{i=0}^{k} G_{i}\right)=\left\{x \in G:\left|x_{I} / 2^{n / 2}\right| \leqslant \Psi(n,|I|), 0 \leqslant|I| \leqslant k\right\} .
\end{aligned}
$$

Далее доказательство разобьем на леммы, дающие оценки $\varphi_{\xi}\left(x / 2^{n / 2-1}\right)$ во введенных областях. В следующей лемме и везде далее все неравенства выполняются начиная с некоторого $n_{0}$.

Лемма 1. Если $x \in G_{i}$ для некоторого $i \in\{0,1, \ldots, k\}$, то не менее чем $\partial л я 2^{n-i}$ векторов $\alpha$ из $V_{n}$ выполняется неравенство

$$
\left|\varphi_{\alpha}\left(x / 2^{n / 2-1}\right)\right| \leqslant 1-\left(\Psi(n, i) / 2^{k+i+1}\right)^{2} .
$$

Доказательство. Если для $y=x / 2^{n / 2}$ спрсведливо равенство

$$
(\beta(\alpha), y)=\rho(\alpha)+\pi t(\alpha)
$$

где $|\rho(\alpha)| \leqslant \pi / 2, t(\alpha)$ - целое число, то

$$
\left|\varphi_{\alpha}\left(x / 2^{n / 2-1}\right)\right|=|\cos (\beta(\alpha), y)|=\cos \rho(\alpha) \leqslant 1-(\rho(\alpha) / 2)^{2} .
$$

Без ограничения общности можно считать, что $\left|y_{I}\right|>\Psi(n, i)$ для $I=\{1, \ldots, i\}$. Разобьем $V_{n}$ на $2^{n-i}$ групп векторов с одинаковыми последними $n-i$ координатами. Достаточно доказать, что в группе с произвольным вектором $\gamma$ последних $n-i$ координат найдется вектор $\alpha$, для которого

$$
|\rho(\alpha)| \geqslant \Psi(n, i) / 2^{k+i}
$$

Рассмотрим величины

$$
\begin{aligned}
p_{0}\left(\alpha_{1}, \ldots, \alpha_{i}\right) & =\left(\beta\left(\alpha_{1}, \ldots, \alpha_{i}, \gamma\right), y\right)=\rho_{0}\left(\alpha_{1}, \ldots, \alpha_{i}\right)+\pi t_{0}\left(\alpha_{1}, \ldots, \alpha_{i}\right) \\
p_{1}\left(\alpha_{2}, \ldots, \alpha_{i}\right) & =\rho_{0}\left(1, \alpha_{2}, \ldots, \alpha_{i}\right)-\rho_{0}\left(0, \alpha_{2}, \ldots, \alpha_{i}\right) \\
\ldots & \\
p_{i}(\varnothing) & =\rho_{i-1}(1)-\rho_{i-1}(0)=\rho_{i}()+\pi t_{i}(\varnothing)
\end{aligned}
$$

где $\left|\rho_{j}(\cdot)\right| \leqslant \pi / 2$ и $t_{j}(\cdot)$ - целые числа для всех $j \in\{0, \ldots, i\}$.

Предположим противное: пусть для всех $\alpha_{1}, \ldots, \alpha_{i}$

$$
\left|\rho_{0}\left(\alpha_{1}, \ldots, \alpha_{i}\right)\right|<\Psi(n, i) / 2^{k+i} .
$$


С одной стороны, $\rho_{i}(\varnothing)$ есть линейная комбинация величин $\rho_{0}\left(\alpha_{1}, \ldots, \alpha_{i}\right)$ с коэффициентами \pm 1, и тогда $\left|\rho_{i}(\varnothing)\right|<\Psi(n, i) / 2^{k}$. С другой стороны,

$$
\begin{aligned}
p_{1}\left(\alpha_{2}, \ldots, \alpha_{i}\right)= & \sum_{I}\left(\beta_{I}\left(1, \alpha_{2}, \ldots, \alpha_{i}, \gamma\right)-\beta_{I}\left(0, \dot{\alpha}_{2}, \ldots, \alpha_{i}, \gamma\right)\right) y_{I} \\
= & \sum_{I \supset\{1\}} \beta_{I}\left(1, \alpha_{2}, \ldots, \alpha_{i}, \gamma\right) y_{I} \\
& \ldots \\
p_{i}(\varnothing)= & \sum_{I \supset\{1, \ldots, i\}} \beta_{I}(1, \ldots, 1, \gamma) y_{I} \\
& \quad+y_{\{1, \ldots, i\}}+\sum_{I \supset\{1, \ldots, i\},|I|>i} \beta_{I}(1, \ldots, 1, \gamma) y_{I}
\end{aligned}
$$

Поскольку $\Psi(n, i)<\left|y_{\{1, \ldots, i\}}\right|<\pi / 2$ и модуль последней суммы не превосходит величины

$$
\begin{aligned}
\sum_{j=1}^{k-i}\left(\begin{array}{c}
n-i \\
j
\end{array}\right) /\left(\left(\begin{array}{c}
n \\
i+j
\end{array}\right) 2^{-n / 3-k(i+j)}\right) & \left.\leqslant 2^{-n / 3-k i} /\left(\begin{array}{c}
n \\
i
\end{array}\right) 2^{-k} \sum_{j=1}^{k-i}\left(\begin{array}{c}
i+j \\
j
\end{array}\right)\right) \\
& \leqslant \Psi(n, i) 2^{-k} \sum_{j=1} k\left(\begin{array}{l}
k \\
j
\end{array}\right) \\
& =\Psi(n, i)\left(1-2^{-k}\right)
\end{aligned}
$$

справедлива оценка

$$
\Psi(n, i) 2^{-k}<\left|p_{i}()\right| \leqslant \pi / 2+\Psi(n, i)<\pi-\Psi(n, i) / 2^{k} .
$$

Следовательно, $\left|p_{i}(\varnothing)\right|>\Psi(n, i) / 2^{k}$, что противоречит оценке, полученной из предположения. Лемма доказана.

Таким образом, для $x \in G_{i}$

$$
\begin{aligned}
\left|\varphi_{\xi}\left(x / 2^{n / 2-1}\right)\right| & \leqslant \exp \left(-2^{n-i}\left(\Psi(n, i) / 2^{k+i+1}\right)^{2}\right) \\
& =\exp \left(-2^{n / 3-2 k i-3 i-2} /\left(\begin{array}{l}
n \\
i
\end{array}\right)\right) \\
& \leqslant \exp \left(-2^{n / 3-2 k^{2}-5 k-2} /\left(\begin{array}{l}
n \\
k
\end{array}\right)\right) .
\end{aligned}
$$

Так как $k^{2}=o(n)$, для всех $x \in G \backslash G^{*}$

$$
\left|\varphi_{\xi}\left(x / 2^{n / 2-1}\right)\right| \leqslant \exp \left(-2^{n / 4}\right) .
$$

Лемма 2. Для $x \in G^{*}$ все величины $d_{\alpha}=\left(\beta(\alpha), x / 2^{n / 2}\right)$ по модулю не превосходят $2^{1-n / 3}$ и характеристическая функция может быть представлена в виде

$$
\varphi_{\xi}\left(x / 2^{n / 2-1}\right)=\exp \left(-\frac{1}{2} x^{T} Q x\left(1-\theta(x) \max _{\alpha \in V_{n}} d_{\alpha}^{2}\right)\right),
$$


где $Q-$ ковариационная матрича сл.в. $(\bar{w}-\mathbf{E} \bar{w}) / 2^{n / 2-1}, 0 \leqslant \theta(x) \leqslant 1 / 2, u$

$$
x^{T} Q x=\sum_{I}\left(\sum_{J \supset I} x_{J} / 2^{|J|}\right)^{2} .
$$

Доказательство. Очевидно, что

$$
\begin{aligned}
\left|d_{\alpha}\right| & \leqslant \sum_{i=0}^{k}\left(\begin{array}{c}
n \\
i
\end{array}\right) \Psi(n, i) \leqslant 2^{-n / 3}\left(1-2^{-k}\right) \leqslant 2^{1-n / 3} \\
\cos d_{\alpha} & =1-d_{\alpha}^{2} / 2+\theta_{1}(x, \alpha) d_{\alpha}^{4}, \quad 0 \leqslant \theta_{1}(x, \alpha) \leqslant 1 / 4 ! .
\end{aligned}
$$

Отсюда,

$$
\begin{aligned}
\varphi_{\alpha}\left(x / 2^{n / 2-1}\right) & =\cos d_{\alpha}=\exp \left(\ln \left(1-\left(d_{\alpha}^{2} / 2-\theta_{1} d_{\alpha}^{4}\right)\right)\right) \\
& =\exp \left(-d_{\alpha}^{2} / 2+\theta_{1} d_{\alpha}^{4}+\sum_{i=2}^{\infty}\left(d_{\alpha}^{2} / 2-\theta_{1} d_{\alpha}^{4}\right)^{i} / i\right) \\
& =\exp \left(-d_{\alpha}^{2} / 2+\theta_{2} d_{\alpha}^{4}\right)=\exp \left(-d_{\alpha}^{2} / 2\left(1-2 \theta_{2} d_{\alpha}^{2}\right)\right)
\end{aligned}
$$

где $0 \leqslant \theta_{2}=\theta_{2}(x, \alpha) \leqslant 1 / 4 !+1 /\left(8\left(\left(1-d_{\alpha}^{2} / 2\right)\right)<1 / 4\right.$. Далее,

$$
\begin{aligned}
\sum_{\alpha} d_{\alpha}^{2} / 2 & =\sum_{\alpha} 2^{-n}\left(\sum_{J} \beta_{J}(\alpha) x_{J} \sum_{K} \beta_{K}(\alpha) x_{K}\right) \\
& =\sum_{J, K} x_{J} x_{K} / 2^{|J \cup K|}=\sum_{J, K} x_{J} x_{K} / 2^{|J|+|K|} \sum_{I \subset J \cap K} 1 \\
& =\sum_{I}\left(\sum_{J \supset I} x_{J} / 2^{|J|} \sum_{K \supset I} x_{K} / 2^{|K|}\right)=\sum_{I}\left(\sum_{J \supset I} x_{J} / 2^{|J|}\right)^{2}
\end{aligned}
$$

Лемма доказана.

Из леммы 3 следует, что для всех $x \in G^{*}$

$$
\left|\varphi_{\xi}\left(x / 2^{n / 2-1}\right)\right| \leqslant \exp \left(-\frac{1-2^{n / 3}}{2} x^{T} Q x\right) .
$$

Рассмотрим далее область

$$
G^{* *}=\left\{x \in G:\left|x_{I}\right| \leqslant 2^{|I|} \sqrt{n(n+1) \ln 4}, 0 \leqslant|I| \leqslant k\right\},
$$

которая содержится в $G^{*}$. Для $x \in G^{* *}$

$$
\begin{aligned}
d_{\alpha}^{2}(x) & \leqslant 2^{-n}\left(\sum_{i=0}^{k}\left(\begin{array}{l}
n \\
i
\end{array}\right) 2^{|I|} \sqrt{n(n+1) \ln 4}\right)^{2} \\
& \leqslant 2^{-n}\left(M(n, k) 2^{k} O(n)\right)^{2}=O\left(n^{2 k+2} / 2^{n}\right) \\
x^{T} Q x & \leqslant \sum_{i=0}^{k}\left(\begin{array}{c}
n \\
i
\end{array}\right)\left(\sum_{t=0}^{k}\left(\begin{array}{l}
n \\
t
\end{array}\right) \sqrt{n(n+1) \ln 4}\right)^{2}=O\left(n^{3 k+2}\right) .
\end{aligned}
$$


Здесь использованы оценки

$$
\begin{gathered}
M(n, k) /\left(\begin{array}{l}
n \\
k
\end{array}\right)-1=\sum_{i=0}^{k-1}\left(\begin{array}{l}
n \\
i
\end{array}\right) /\left(\begin{array}{l}
n \\
k
\end{array}\right) \\
\left.2^{k}\left(\begin{array}{l}
n \\
k
\end{array}\right)=O\left(\begin{array}{c}
n \\
k-1
\end{array}\right) /\left(\begin{array}{l}
n \\
k
\end{array}\right)=O\left(k^{2}\right) . n\right)=o(1)
\end{gathered}
$$

Следовательно, равномерно по $x \in G^{*}$

$$
\begin{aligned}
\varphi_{\xi}\left(x / 2^{n / 2-1}\right) & =\exp \left(-\frac{1}{2} x^{T} Q x\right) \exp \left(O\left(n^{5 k+4} / 2^{n}\right)\right) \\
& =\exp \left(-\frac{1}{2} x^{T} Q x\right)\left(1+O\left(n^{5 k+4} / 2^{n}\right)\right) .
\end{aligned}
$$

Лемма 3. Справедливы равенства

$$
\begin{aligned}
\operatorname{det} Q & =\exp _{2}\left(-2 \sum_{i=0}^{k} i\left(\begin{array}{c}
n \\
i
\end{array}\right)\right)=\exp _{2}\left((n-k)\left(\begin{array}{l}
n \\
k
\end{array}\right)-n M(n, k)\right), \\
z^{T} Q^{-1} z & =\sum_{I}\left(2^{|I|} z_{I}-\sum_{i \in I} 2^{|I|-1} z_{I \backslash\{i\}}\right)^{2} .
\end{aligned}
$$

Доказателъство. Положим

$$
y_{I}=\sum_{J \supset I} x_{J} / 2^{|J|}
$$

Согласно лемме 3 для матрицы $A$ перехода от $x$ к $y(y=A x)$ справедливы равенства

$$
x^{T} Q x=y^{T}\left(A^{-1}\right)^{T} Q A^{-1} y=y^{T} y
$$

то есть

$$
Q=A^{T} A, \quad Q^{-1}=A^{-1}\left(A^{-1}\right)^{T} .
$$

Элемент $A_{I J}$ равен $2^{-|J|}$, если $I \subset J$, и нулю в противном случае, поэтому $A-$ верхнетреугольная матрица и

$$
\operatorname{det} Q=(\operatorname{det} A)^{2}=\exp _{2}\left(-2 \sum_{i=0}^{k} i\left(\begin{array}{c}
n \\
i
\end{array}\right)\right)
$$

Для доказательства первого утверждения леммы остается заметить, что

$$
\begin{aligned}
M(n, k) & =\sum_{i=0}^{k}\left(\left(\begin{array}{c}
n-1 \\
i-1
\end{array}\right)+\left(\begin{array}{c}
n-1 \\
i
\end{array}\right)\right)=\sum_{i=0}^{k-1}\left(\begin{array}{c}
n-1 \\
i
\end{array}\right)+\sum_{i=0}^{k}\left(\begin{array}{c}
n-1 \\
i
\end{array}\right) \\
& =2 M(n-1, k-1)+\left(\begin{array}{c}
n-1 \\
k
\end{array}\right), \\
\sum_{i=0}^{k} i\left(\begin{array}{c}
n \\
i
\end{array}\right) & =n M(n-1, k-1), \quad n\left(\begin{array}{c}
n-1 \\
k
\end{array}\right)=(n-k)\left(\begin{array}{l}
n \\
k
\end{array}\right) .
\end{aligned}
$$


Чтобы найти $A^{-1}$, выразим $x$ через $y$. При $|I|=k$ по определению $x_{I} / 2^{|I|}=y_{I}$. Докажем индукцией по $|I|$ от $k-1$ до 0 равенство

$$
x_{I} / 2^{|I|}=y_{I}-\sum_{i \notin I} y_{I \cup\{i\}} .
$$

При $|I|=k-1$ оно верно, так как

$$
y_{I}=x_{I} / 2^{k-1}+\sum_{i \notin I} x_{I \cup\{i\}} / 2^{k}=x_{I} / 2^{k-1}+\sum_{i \notin I} y_{I \cup\{i\}} .
$$

Предположим, что равенство верно при $|I|=l+1, \ldots, k-1$ и докажем его для $|I|=l$ :

$$
\begin{aligned}
y_{I} & =x_{I} / 2^{l}+\sum_{J \supset I, J \neq I}\left(y_{J}-\sum_{i \notin J} y_{J \cup\{i\}}\right) \\
& =x_{I} / 2^{l}+\sum_{J \supset I, J \neq I} y_{J}-\sum_{K \supset I,|K| \geqslant|I|+2} y_{K} \\
& =x_{I} / 2^{l}+\sum_{J \supset I,|J|=|I|+1} y_{J} .
\end{aligned}
$$

Таким образом,

$$
\begin{aligned}
&\left(A^{-1}\right)_{I J}= \begin{cases}2^{|I|}, & \text { если } I=J, \\
-2^{|I|}, & \text { если } J=I \cup\{i\}, i \notin I, \\
0 & \text { в остальных случаях, }\end{cases} \\
&\left(\left(A^{-1}\right)^{T}\right)_{I J}= \begin{cases}2^{|I|}, & \text { если } I=J, \\
-2^{|J|}, & \text { если } J=I \backslash\{i\}, i \in I, \\
0 & \text { в остальных случаях. }\end{cases}
\end{aligned}
$$

Отсюда,

$$
z^{T} Q^{-1} z=z^{T} A^{-1}\left(A^{-1}\right)^{T} z=\sum_{I}\left(2^{|I|} z_{I}-\sum_{i \in I} 2^{|I|-1} z_{I \backslash\{I\}}\right)^{2}
$$

Лемма доказана.

Разобьем интеграл в (1) на сумму интегралов по областям $G \backslash G^{*}, G^{*} \backslash G^{* *}, G^{* *}$ и обозначим их соответственно через $J_{1}, J_{2}, J_{3}$. Из (2)-(3) следует, что

$$
\begin{aligned}
& \left|J_{1}\right| \leqslant\left(\pi 2^{n / 2}\right)^{M} \exp \left(-2^{n / 4}\right) \\
& \left|J_{2}\right| \leqslant \sqrt{(2 \pi)^{M} / \operatorname{det}\left(\left(1-2^{1-n / 3}\right) Q\right) \mathbf{P}\left(\eta \in G^{*} \backslash G^{* *}\right),}
\end{aligned}
$$

где $\eta$ - нормально распределенный вектор с параметрами $\left(\overline{0},\left(\left(1-2^{1-n / 3}\right) Q\right)^{-1}\right)$. Поскольку (см. доказательство леммы 4)

$$
\left(Q^{-1}\right)_{I I}=\sum_{I}\left(A^{-1}\right)_{I J}^{2} \leqslant 2^{2|I|}(1+n-|I|),
$$


справедливы оценки

$$
\begin{aligned}
\mathbf{P}\left(\left|\eta_{I}\right|>2^{|I|} \sqrt{n+1} \sqrt{n \ln 4}\right) & \leqslant \frac{2}{\sqrt{2 \pi}} \int_{\sqrt{n \ln 4\left(1-2^{1-n / 3}\right)}}^{\infty} \exp \left(-t^{2} / 2\right) d t \\
& \leqslant \frac{2 \exp \left(-n \ln 2\left(1-2^{1-n / 3}\right)\right)}{\sqrt{2 \pi n \ln 4\left(1-2^{1-n / 3}\right)}}=o\left(2^{-n}\right) \\
\mathbf{P}\left(\eta \in G^{*} \backslash G^{* *}\right) & \leqslant \sum_{I} \mathbf{P}\left(\left|\eta_{I}\right|>2^{|I|} \sqrt{(n+1) n \ln 4}\right)=o\left(n^{k} / 2^{n}\right) .
\end{aligned}
$$

Учитывая, что

$$
\operatorname{det}\left(\left(1-2^{1-n / 3}\right) Q\right)=\left(1-2^{1-n / 3}\right)^{M} \operatorname{det} Q=(1+o(1)) \operatorname{det} Q
$$

получаем оценку

$$
\left|J_{2}\right|=\sqrt{(2 \pi)^{M} / \operatorname{det} Q} O\left(n^{k} / 2^{n}\right)
$$

Согласно (4),

$$
J_{3}=J_{4}-J_{5}+J_{6}
$$

где аналогично оценке $J_{2}$

$$
\begin{aligned}
& J_{4}=\int_{R^{M}} \exp \left(-\frac{1}{2} x^{T} Q x\right) \exp (-i(x, z)) d x=\sqrt{(2 \pi)^{M} / \operatorname{det} Q} \exp \left(-\frac{1}{2} z^{T} Q^{-1} z\right), \\
& J_{5}=\int_{R^{M} \backslash G^{* *}} \exp \left(-\frac{1}{2} x^{T} Q x\right) \exp (-i(x, z)) d x=\sqrt{(2 \pi)^{M} / \operatorname{det} Q} O\left(n^{k} / 2^{n}\right)
\end{aligned}
$$

И

$$
J_{6}=\int_{G^{* *}} \exp \left(-\frac{1}{2} x^{T} Q x\right) O\left(n^{5 k+4} / 2^{n}\right) d x=\sqrt{(2 \pi)^{M} / \operatorname{det} Q} O\left(n^{5 k+4} / 2^{n}\right) .
$$

Из этих оценок, формул (1), (5), (6) и леммы 4 находим, что

$$
\begin{aligned}
\left(\pi 2^{n / 2}\right)^{M} \mathbf{P}\left(\xi=z 2^{n / 2-1}\right)= & \sqrt{(2 \pi)^{M} / \operatorname{det} Q}\left(\exp \left(-\frac{1}{2} z^{T} Q^{-1} z\right)\right. \\
& \left.+O\left(n^{k} / 2^{n}\right)+O\left(n^{5 k+4} / 2^{n}\right)\right)+\left(\pi 2^{n / 2}\right)^{M} \exp \left(-2^{n / 4}\right) \\
= & \frac{\exp \left(-(1 / 2) \bar{z}^{T} Q^{-1} \bar{z}\right)+O\left(n^{5 k+4} / 2^{n}\right)}{\exp _{2}\left(\left(\begin{array}{l}
n \\
k
\end{array}\right)(n-k) / 2-n M / 2-M / 2 \log _{2} 2 \pi\right)} .
\end{aligned}
$$

Теорема 1 доказана.

Согласно формуле (1) работы [1], критерием принадлежности функции $f$ классу $K(n, k)$ является условие

$$
\exists r \in\left\{-2^{n-k-1}, \ldots, 2^{n-k-1}\right\}: \forall 0 \leqslant|I| \leqslant k \quad w_{I}=\mathbf{E} w_{I}+. r 2^{k-|I|} .
$$

Поэтому

$$
\frac{|R(n, k)|}{\left|B_{n}\right|}=\mathbf{P}\{f \in R(n, k)\}=\mathbf{P}\{\bar{w}=\mathbf{E} \bar{w}\}
$$

и справедлива следующая асимптотическая формула для числа $(n, 1, k)$-устойчивых функций. 
Следствие 1. Ecли $n \rightarrow \infty u k(n)=o(\sqrt{n}), m o$

$$
|R(n, k)| \sim \exp _{2}\left(2^{n}-\frac{n-k}{2}\left(\begin{array}{l}
n \\
k
\end{array}\right)-M(n, k) \log _{2} \sqrt{\pi / 2}\right) .
$$

Согласно (7)

$$
\mathbf{P}\{f \in K(n, k)\}=\sum_{r=-2^{n-k-1}}^{2^{n-k-1}} \mathbf{P}\left\{\bar{w}=r \bar{z} 2^{n / 2-1}\right\}
$$

где $\bar{z}$ - вектор с компонентами $z_{I}=2^{k-|I|-n / 2+1}, 0 \leqslant|I| \leqslant k$.

Для числа корреляционно-иммунных порядка $k$ функций от $n$ переменных справедливо следующее утверждение.

Следствие 2. Если $n \rightarrow \infty u k(n)=o(\sqrt{n})$, mo

$$
\begin{aligned}
|K(n, k)| & \sim \frac{|R(n, k)| \sqrt{\pi / 2} 2^{n / 2-k}}{\left(1+\sum_{i=2}^{k}(i-1)^{2}\left(\begin{array}{c}
n \\
i
\end{array}\right)\right)^{1 / 2}} \\
& \sim \frac{\exp _{2}\left(2^{n}-\left(\begin{array}{c}
n \\
k
\end{array}\right)(n-k) / 2-(M(n, k)-1) \log _{2} \sqrt{\pi / 2}+n / 2-k\right)}{\left(1+\sum_{i=2}^{k}(i-1)^{2}\left(\begin{array}{c}
n \\
i
\end{array}\right)\right)^{1 / 2}} .
\end{aligned}
$$

Доказательство. Из (8) и теоремы 1 следует, что

$$
\begin{aligned}
\frac{|K(n, k)|}{\left|B_{n}\right|} & =\sum_{r=-2^{n-k-1}}^{2^{n-k-1}} \frac{|R(n, k)|}{\left|B_{n}\right|}\left(\exp \left(-\frac{r^{2}}{2} \bar{z}^{T} Q^{-1} \bar{z}\right)+O\left(n^{5 k+4} / 2^{n}\right)\right), \\
\bar{z}^{T} Q^{-1} \bar{z} & =2^{-n+2} \sum_{I}\left(2^{|I|} 2^{k-|I|}-\sum_{i \in I} 2^{|I|-1} 2^{k-|I|-1}\right)^{2} \\
& =2^{-n+2} \sum_{I}\left(2^{k}-|I| 2^{k}\right)^{2}=2^{2 k-n+2} \sum_{i=0}^{k}\left(\begin{array}{c}
n \\
i
\end{array}\right)(1-i)^{2}=2^{2 k-n+2},
\end{aligned}
$$

где

$$
S=1+\sum_{i=2}^{k}(i-1)^{2}\left(\begin{array}{c}
n \\
i
\end{array}\right)
$$

Тогда

$$
|K(n, k)|=|R(n, k)|\left(\frac{2^{n / 2-k-1}}{\sqrt{S}} \sum_{r} \exp \left(-x_{r}^{2} / 2\right)\left(x_{r+1}-x_{r}\right)+o(1)\right),
$$

где $x_{r}=r \sqrt{S} / 2^{n / 2-k-1}$. Сумма в скобках есть интеграл от непрерывной функции по ступенчатой функции в пределах от $-2^{n / 2} / \sqrt{S}$ до $2^{n / 2} / \sqrt{S}$, который при $n \rightarrow \infty$ сходится к интегралу

$$
\int_{-\infty}^{\infty} \exp \left(-t^{2} / 2\right) d t=\sqrt{2 \pi}
$$

Следствие доказано. 
В следующей таблице приведены точные значения $|R(n, k)|,|K(n, k)|$ и приближенные значения, вычисленные по формулам

$$
\begin{aligned}
& |R(n, 1)|^{*}=\pi^{-(n+1) / 2} \exp _{2}\left(2^{n}-\frac{n^{2}-2 n-1}{2}\right) \\
& |R(n, 2)|^{*}=\pi^{-\left(n^{2}+n\right) / 4} \exp _{2}\left(2^{n}-\frac{n^{3}-4 n^{2}+n-2}{4}\right) \\
& |K(n, 1)|^{*}=\pi^{-n / 2} \exp _{2}\left(2^{n}-\frac{n^{2}-3 n+2}{2}\right) \\
& |K(n, 2)|^{*}=\pi^{-\frac{n^{2}+n}{4}} \exp _{2}\left(2^{n}-\frac{n^{3}-4 n^{2}-n+8}{4}\right) / \sqrt{n(n+1) / 2}
\end{aligned}
$$

а также относительные погрешности $\left(x^{*}-x\right) / x$ в процентах.

\begin{tabular}{|c|c|c|c|c|c|c|c|}
\hline$n$ & $k$ & $|R(n, k)|$ & $|R(n, k)|^{*}$ & $\%$ & $|K(n, k)|$ & $|K(n, k)|^{*}$ & $\%$ \\
\hline 4 & 1 & 222 & 331,13 & 50 & 648 & 830,02 & $28,1 \%$ \\
\hline 4 & 2 & 10 & 85,44 & 754,4 & 12 & 33,86 & $182,2 \%$ \\
\hline 5 & 1 & 807980 & $1.08 \cdot 10^{6}$ & 33,9 & 314062 & $3,84 \cdot 10^{6}$ & $22,2 \%$ \\
\hline 5 & 2 & 552 & 3536,31 & 540,6 & 1058 & 1618,38 & $53 \%$ \\
\hline
\end{tabular}

Из таблицы видно, что для $k=1$ формулы дают правильный порядок уже при $n=4,5$. Для $k=2$ и данных значений $n$ величина отношения $k^{2} / n$ велика и, видимо, этим в первую очередь объясняется большая величина погрешности.

Заметим, что, используя формулу условной вероятности, мы можем теперь оценивать распределение вектора $\bar{w}(f, n, k)$ при равновероятном выборе $f$ не из всего $B_{n}$, а из множества

$$
\left\{f \in B_{n}: \bar{w}(f, n, l)=\bar{w}_{0}\right\}
$$

при любых $0 \leqslant l<k$ и $\bar{w}_{0} \in Z^{M(n, l)}$. Так, при $l=0$ и $\bar{w}_{0}=(w)$ получаем при $n \rightarrow \infty$ предельное распределение $\bar{w}$ при случайном выборе $f$ из функций веса $w=w(n)$. Для иллюстрации сформулируем еще одно следствие.

Следствие 3. Если $n \rightarrow \infty u k(n)=o(\sqrt{n})$, mo

$$
\begin{aligned}
& \mathbf{P}\{f \in R(n, k+1) \mid f \in R(n, k)\}=\frac{|R(n, k+1)|}{|R(n, k)|} \\
& \sim \exp _{2}\left(-\frac{n-k-1}{2}\left(\begin{array}{c}
n \\
k+1
\end{array}\right)+\frac{n-k}{2}\left(\begin{array}{l}
n \\
k
\end{array}\right)-\left(\begin{array}{c}
n \\
k+1
\end{array}\right) \log _{2} \sqrt{\pi / 2}\right) \\
& =\exp _{2}\left(-\frac{1}{2}\left(\begin{array}{c}
n \\
k+1
\end{array}\right)\left(n-k-1-k-1+\log _{2} \frac{\pi}{2}\right)\right) \\
& =\exp _{2}\left(-\frac{1}{2}\left(\begin{array}{c}
n \\
k+1
\end{array}\right)\left(n-2 k-\log _{2} \frac{8}{\pi}\right)\right) \text {. }
\end{aligned}
$$

\section{3. ЛПТ для распределения части спектра}

Многие важные характеристики двоичных функций определяются частью спектра. В работе [7] доказано, что $f \in K(n, k)$ в том и только том случае, когда $F_{I}=0$ для всех $I, 1 \leqslant|I| \leqslant k$. Следовательно, $f \in R(n, k)$ в том и только том случае, когда 
$F_{I}=0$ для всех $I, 0 \leqslant|I| \leqslant k$. В статье [8] этот критерий обобщен для функций $f: G F(q)^{n} \rightarrow G F(q)^{m}$.

В двоичном случае этот факт вытекает из однозначной связи векторов $\bar{w}(f, n, k)$ и $\bar{F}(f, n, k)$, которая, в свою очередь следует из известных формул связи весов подфункций функции и ее спектральных коэффициентов. Дадим простое доказательство формул связи. Заметим, что $(-1)^{a}=1-2 a$ для $a=0,1$ и

$$
\begin{aligned}
a_{1} \oplus \ldots \oplus a_{m} & =\frac{1}{2}\left(1-(-1)^{a_{1}+\ldots+a_{m}}\right)=\frac{1}{2}\left(1-\prod_{j=1}^{m}\left(1-2 a_{j}\right)\right) \\
& =\frac{1}{2}\left(1-\sum_{J \subset\{1, \ldots, m\}}(-2)^{|J|} a_{j_{1}} \ldots a_{j_{|J|} \mid}\right) \\
& =\sum_{\varnothing \neq J \subset\{1, \ldots, m\}}(-2)^{|J|-1} a_{j_{1}} \ldots a_{j_{|J|} .}
\end{aligned}
$$

Отсюда следует, что

$$
\begin{aligned}
\| f\left(x_{1}, \ldots, x_{n}\right) & \oplus x_{i_{1}} \ldots \oplus x_{i_{|I|} \mid} \| \\
& =\sum_{x \in V_{n}}\left(\sum_{\varnothing \neq J \subset I}(-2)^{|J|-1} x_{j_{1}} \ldots x_{j_{|J|}}+\sum_{J \subset I}(-2)^{|J|} x_{j_{1}} \ldots x_{j_{|J|}} f(x)\right) \\
& =\sum_{\varnothing \neq J \subset I}(-2)^{|J|-1} 2^{n-|J|}+\sum_{J \subset I}(-2)^{|J|} \sum_{x \in V_{n}} x_{j_{1}} \ldots x_{j_{|J|}} f(x) \\
& =2^{n-1} \sum_{\varnothing \neq J \subset I}\left((-1)^{|J|-1}-1+1\right)+\sum_{J \subset I}(-2)^{|J|} w_{J} \\
& =2^{n-1}+\sum_{J \subset I}(-2)^{|J|} w_{J}, \\
F_{I} & =-\sum_{J \subset I}(-2)^{|J|} w_{J}=\sum_{J \subset I}(-1)^{|J|}\left(2^{n-1}-2^{|J|} w_{J}\right) .
\end{aligned}
$$

Тогда по формуле обращения Мебиуса

$$
2^{n-1}-2^{|I|} w_{I}=\sum_{J \subset I}(-1)^{|J|} F_{J} w_{I}-2^{n-|I|-1}=2^{-|I|} \sum_{J \subset I}(-1)^{|J|+1} F_{J} .
$$

Из (11) легко получить отношение сравнимости для спектральных коэффициентов, именно, для всех $1 \leqslant|I| \leqslant k$

$$
F_{I} \equiv \sum_{J \subset I, J \neq I}(-1)^{|I \backslash J|-1} F_{J} \quad\left(\bmod 2^{|I|}\right)
$$

При $k=1$ это сравнение равносильно одинаковой четности компонент $\bar{F}$. Таким образом, из теоремы 1 и формул связи (10)-(11) векторов $\bar{w}$ и $\bar{F}$ можно вывести асимптотическую формулу для вероятностей $\mathbf{P}(\bar{F}=\bar{a})$ для целочисленных векторов $\bar{a}$ длины $M(n, k)$, удовлетворяющих условиям (12). 
Теорема 2. Пусть $n \rightarrow \infty u k(n)=o(\sqrt{n})$. Тогда равномерно относителъно челочисленных векторов $\bar{a}$ длины $M(n, k)$, удовлетворяющих условиям (12) сравнимости компонент $a_{I}$, справедливо представление

$$
\mathbf{P}\{\bar{F}=\bar{a}\}=\frac{\exp \left(-U_{k}(\bar{a}) / 2^{n-1}\right)+O\left(n^{5 k+4} / 2^{n}\right)}{\exp _{2}\left(\left(\begin{array}{l}
n \\
k
\end{array}\right)(n-k) / 2+M(n, k) \log _{2} \sqrt{\pi / 2}\right)},
$$

гдe

$$
U_{k}(\bar{a})=\sum_{0 \leqslant|I| \leqslant k}\left(\sum_{J \subset I}(-1)^{|J|}(|I|-|J|-1) a_{J}\right)^{2}
$$

В частности,

$$
\begin{aligned}
& U_{1}(\bar{a})=\sum_{|I| \leqslant 1} a_{I}^{2}, \\
& U_{2}(\bar{a})=\sum_{|I| \leqslant 1} a_{I}^{2}+\sum_{|I|=2}\left(a_{\varnothing}-a_{I}\right)^{2}, \\
& U_{3}(\bar{a})=\sum_{|I| \leqslant 1} a_{I}^{2}+\sum_{|I|=2}\left(a_{\varnothing}-a_{I}\right)^{2}+\sum_{|I|=3}\left(2 a_{\varnothing}-a_{\left\{i_{1}\right\}}-a_{\left\{i_{2}\right\}}-a_{\left\{i_{3}\right\}}+a_{\left\{i_{1}, i_{2}, i_{3}\right\}}\right)^{2} .
\end{aligned}
$$

Доказательство. Из теоремы 1 и формул (10)-(11) получаем, что

$$
\mathbf{P}\{\bar{F}=\bar{a}\}=\mathbf{P}\{\bar{w}-\mathbf{E} \bar{w}=\bar{z}(\bar{a})\}=\frac{\exp \left(-(1 / 2) \bar{z}^{T}(\bar{a}) Q(n, k)^{-1} \bar{z}(\bar{a}) / 2^{n-2}\right)+O\left(n^{5 k+4} / 2^{n}\right)}{\left.\exp _{2}\left(\begin{array}{l}
n \\
k
\end{array}\right)(n-k) / 2+M(n, k) \log _{2} \sqrt{\pi / 2}\right)}
$$

равномерно относительно $\bar{a}$ таких, что вектор $\bar{z}(\bar{a})$ с компонентами

$$
z_{I}(\bar{a})=2^{-|I|} \sum_{J \subset I, J \neq I}(-1)^{|J|+1} a_{J}
$$

целочисленный, что равносильно условию (12). Остается заметить, что

$$
\bar{z}^{T}(\bar{a}) Q(n, k)^{-1} \bar{z}(\bar{a})=U_{k}(\bar{a}),
$$

поскольку (см. лемму 4)

$$
\begin{aligned}
2^{|I|} z_{|I|}-\sum_{i \in I} 2^{|I|-1} z_{I \backslash\{i\}} & =\sum_{J \subset I}(-1)^{|J|+1} a_{J}-\sum_{K \subset I,|K|=|I|-1} \sum_{J \subset K}(-1)^{|J|+1} a_{J} \\
& =\sum_{J \subset I}(-1)^{|J|+1} a_{J}(1-|\{K: J \subset K \subset I,|K|=|I|-1\}|) \\
& =\sum_{J \subset I}(-1)^{|J|}(|I \backslash J|-1) a_{J} .
\end{aligned}
$$

Теорема доказана.

В работе Б.В. Рязанова [4] была доказана (а в [5] обобщена) следующая ЛПТ для распределения части спектра. Согласно формуле (17) из [4] при любом фиксированном $r \geqslant 1$ и $n \rightarrow \infty$ асимптотическое равенство

$$
\mathbf{P}\left\{F_{I_{j}}=a_{j}, 1 \leqslant j \leqslant r\right\}=\frac{\exp \left(-\sum_{j=1}^{r} a_{j}^{2} / 2^{n-1}\right)+O\left(2^{-n / 2}\right)}{\exp _{2}\left(n r / 2-r+1+r \log _{2} \sqrt{\pi / 2}\right)}
$$


выполняется равномерно по всем точкам $\bar{a}=\left(a_{1}, \ldots, a_{r}\right)$ некоторой целочисленной решетки и наборам $\left(I_{1}, \ldots, I_{r}\right)$ таким, что соответствующие им векторы $e_{I_{1}}, \ldots, e_{I_{r}}$ $\left(I-\right.$ множество единичных координат вектора $\left.e_{I} \in V_{n}\right)$ линейно независимы.

Теорема 2 и ЛПТ из [4] характеризуют поведение непересекающихся множеств наборов спектральных коэффициентов. Тем не менее, если рассматривать предельное распределение первых $M(n, 1)$ спектральных коэффициентов, то главные члены в теореме 2 и формуле (13) при $r=n+1$ одинаковы. Но на этом сходство оканчивается: при всех $k \geqslant 2$ и квадратичная форма $U_{k}(\bar{a})$, и выражение в знаменателе отличаются от соответствующих выражений в (13).

Автор благодарен А.С. Амбросимову за постановку задачи и внимание к работе.

\section{Список литературы}

1. Денисов О.В., Асимптотическая формула для числа корреляционно-иммунных порядка $k$ булевых функций. Дискретная математика (1991) 3, №2, 25-46.

2. Зуев Ю.А., Комбинаторно-вероятностные и геометрические методы в пороговой логике. Дискретная математика (1991) 3, №2, 47-57.

3. Кузнецов Ю.В., Шкарин С.А., Коды Рида-Маллера (обзор публикаций). Матем. вопросы кибернетики (1996), №6, 51-80.

4. Рязанов Б.В., О распределении спектральной сложности булевых функций. Дискретнал математика (1994) 6, №2, 111-129.

5. Рязанов Б.В., Чечета С.И., О приближении случайной булевой функции множеством квадратичных форм. Дискретная математика (1995) 7, №3, 129-145.

6. Chow C.K., On the characterization of threshold functions. Minimization of Boolean Functions and Logical Design. Switching Circuit Theory and Logical Design. AIEE Special Publication (1961) 134, 34-38.

7. Guo-Zhen X., Massey J.L., A spectral characterization of correlation-immune icombining functions. IEEE Trans. Information Theory (1988) 34, 569-571.

8. Gopalakrishnan K., Stinson D.R., Three characterization of non-binary correlation-immune and resilient functions. Designs, Codes and Cryptography (1995) 5, 241-251.

Статья поступила 09.11.1999. 